\title{
Performances zootechniques des caprins au Niger : étude comparative de la chèvre rousse de Maradi et de la chèvre à robe noire dans la zone de Maradi
}

\author{
H. Marichatou ${ }^{1 *}$ L. Mamane ${ }^{2}$ M. Banoin ${ }^{1}$ G. Baril ${ }^{3}$
}

\section{Mots-clés}

Caprin - Chèvre rousse de Maradi Chevreau - Performance de reproduction - Croissance - Niger.

\begin{abstract}
Résumé
L'objectif principal de cette étude a été non seulement de contribuer à la connaissance de la chèvre rousse de Maradi, mais également de comparer les paramètres de reproduction, le poids adulte, les mensurations et la croissance de la chèvre noire à ceux de la rousse. Des enquêtes verbales et rétrospectives ont été réalisées pour trois lots de chèvres (noires et rousses) : 106 ayant eu 3 à 4 mises bas, 97 ayant eu deux mises bas et 87 primipares. Sept mensurations ainsi que les poids adultes ont également été relevés sur 125 femelles rousses et noires de 4 ans \pm 3 mois. L'évolution pondérale de 38 cabris noirs et roux en milieu villageois a été suivie de la naissance à deux mois. L'analyse statistique des données sur les deux robes en milieu villageois a montré des résultats globalement analogues. Néanmoins, la prolificité a toujours été plus élevée chez les chèvres à robe rousse que chez les chèvres à robe noire quelle qu'ait été la parité.
\end{abstract}

\section{INTRODUCTION}

Au Niger, l'élevage contribue pour 12 p. 100 au Produit intérieur brut national et pour 31 p. 100 au Pib agricole (15). L'effectif des caprins est important (6037000 têtes en 1996), avec une hausse croissante depuis 1980 en partie attribuable à l'adaptation de l'espèce caprine aux conditions souvent défavorables pour les autres espèces animales domestiques, notamment les bovins et les ovins.

\footnotetext{
1. Faculté d'Agronomie, BP 10960, université Abdou Moumouni, Niamey, Niger 2. Direction des Centres de multiplication du bétail, ministère des Ressources animales, Niamey, Niger

3. Inra-Cnrs, Umr 6073, Physiologie de la reproduction et des comportements, 37380 Nouzilly, France

* Auteur pour la correspondance

Cirdes, 01 BP 454, Bobo-Dioulasso 01, Burkina Faso
}

Tél. : (226) 975794 ; fax : (226) 972320 ; e-mail : m.amani@ fasonet.bf
Les caprins se répartissent principalement entre deux races largement métissées : la chèvre sahélienne, adaptée aux longs déplacements, dans la frange nord du Niger, dont la robe comporte le noir, le blanc et le rouge; la chèvre rousse dans le sud du pays, où les agriculteurs associent son élevage à l'agriculture, dont la robe est rousse. A.H. Robinet écrivait en 1967 : « La robe [de la chèvre rousse de Maradi] est homogène, brillante, à reflets acajou [...]. Le mâle présente de manière constante une teinte plus foncée allant jusqu'à l'apparition d'une raie dorsale noire [...]. Les métis ont une robe généralement bariolée ou mélangée de blanc ou de rouge. Les muqueuses visibles sont noires. Tout éclaircissement de la fourrure, apparition de teintes délavées et de poils blancs, marque un recul dans la pureté du type. [Ainsi, parmi les robes acajou, ont pu être distinguées les nuances suivantes :] acajou avec ligne dorsale noire bien nette ; acajou avec quelques poils noirs sans localisation nette ; acajou sans poils noirs autres que ceux des extrémités » (19).

Les aptitudes zootechniques très marquées de la chèvre rousse ont été décrites $(2,17,19,20)$. L'exceptionnelle qualité de sa peau lui a valu une renommée internationale. L'attention particulière qui lui 
est accordée a incité plusieurs actions au niveau national permettant sa diffusion : distribution de caprins roux, mesures législatives très sévères en vue de sa préservation (interdiction d'abattage de femelles rousses, élimination des chèvres bariolées et communes, castration des boucs non roux). En 1965, un Centre d'élevage caprin fut créé avec pour objectifs principaux la sélection et la diffusion de la chèvre rousse de Maradi. Malgré ces énormes efforts, il cohabite dans la même aire géographique une autre chèvre à robe uniformément noire, avec un effectif sans cesse croissant. Son ethnologie n'ayant pas encore été élucidée, aucune étude ne précise ses performances. Seul, un sondage autour du Centre caprin (16) révèle que du point de vue économique les producteurs ne distinguent pas cette chèvre noire de la chèvre rousse. De même, sur les peaux uniformément rousses ou noires, la société nigérienne des cuirs et peaux (Sncp) et celle des tanneries du Niger (Sonitan) affichent les mêmes prix.

Dans le but de mieux connaître la chèvre noire et de déterminer si c'est une variante de la chèvre rousse, dans la présente étude ont été comparés certains paramètres zootechniques d'intérêt économique, notamment sur la reproduction, la croissance des jeunes jusqu'à deux mois et les mensurations à l'âge adulte.

\section{MATERIEL ET METHODES}

\section{Conditions et animaux de l'étude}

Cette enquête transversale a été réalisée chez les éleveurs encadrés par le projet d'Appui à l'élevage de la chèvre rousse, dans 15 villages des arrondissements de Tessaoua, Madarounfa et GuidanRoumdji, du département du Maradi. Cette région est considérée comme le berceau de la chèvre rousse. Les précipitations y varient de $200 \mathrm{~mm}$ au Nord (Dakoro) à $700 \mathrm{~mm}$ au Sud (Madarounfa). Les courbes de température moyennes mensuelles présentent deux maxima, en avril-mai $\left(32{ }^{\circ} \mathrm{C}\right)$ et en octobre $\left(29,5{ }^{\circ} \mathrm{C}\right)$, et une minima en décembre-janvier $\left(22^{\circ} \mathrm{C}\right)$.

Les éleveurs concernés ont été au nombre de 77 dont 26 à GuidanRoumdji, 19 à Madarounfa et 32 à Tessaoua. Les critères de leurs choix ont été : la disponibilité à fournir des renseignements clairs et précis ; la possession d'un troupeau caprin d'un effectif minimal de sept caprins roux ou noirs ; la localisation dans un des villages encadrés par le projet et dans la plupart des cas suivis par les agents du projet. Les conditions d'élevage ont été assez homogènes au niveau des arrondissements.

Les catégories d'animaux qui ont fait l'objet de l'étude ont été (tableau I) : les cabris, de la naissance à deux mois (pour la croissance) ; les primipares (pour l'âge à la première mise bas) ; les chèvres à deux mises bas (pour l'intervalle entre les mises bas); les chèvres adultes de 4 ans \pm 3 mois ayant eu 3 ou 4 mises bas (pour les mensurations, la prolificité).

Concernant l'alimentation, deux périodes ont dû être considérées. La première allait du début de la saison des pluies à la fin des récoltes (juin-novembre), lorsque les caprins étaient gardés au piquet en permanence et nourris au brout, de produits de sarclage et de chaumes. La deuxième correspondait à la saison sèche, lorsque les animaux étaient en divagation ou conduits au pâturage. Au début, les chèvres ont profité largement des résidus de récoltes relayés par la suite par les pâturages naturels (ligneux et herbacés). La supplémentation minérale a été assurée pendant toute l'année dans 96 p. 100 des exploitations. Le son domestique a été introduit en complément dans 30 p. 100 des troupeaux. L'abreuvement s'est fait une à deux fois par jour.

En matière de santé, seuls 21 p. 100 des exploitants vaccinaient leurs troupeaux. La vermifugation non systématique n'était réalisée que chez 13 p. 100 des exploitants.

\section{Analyse des données}

Une enquête rétrospective a été faite pour chaque femelle en vue de reconstituer sa carrière reproductrice : naissance, nombre de mises bas, taille de la portée à chaque mise bas, avortements, etc. Les femelles adultes de 4 ans \pm 3 mois ont fait l'objet de mesures aux mois d'août et septembre, portant sur la taille au garrot, la longueur et la largeur de la tête, la circonférence pectorale, la profondeur de la poitrine, la longueur scapulo-ischiale, la longueur scapulo-iliale, le poids adulte (à l'aide d'un peson de portée allant jusqu'à $100 \mathrm{~kg}$ et de précision à $500 \mathrm{~g}$ près). Les cabris ont été pesés à la naissance, puis suivis à 1 et 2 mois d'âge, à l'aide d'un peson de portée allant jusqu'à $25 \mathrm{~kg}$ et de précision à $100 \mathrm{~g}$ près.

Les données ont été présentées sous forme de moyennes arithmétiques. L'analyse de la variance suivant le test de Fisher et le test de Student au risque de première espèce de 5 p. 100 a été utilisée pour tester les éventuels effets de la race, du milieu, etc.

\section{RESULTATS ET DISCUSSION}

\section{Paramètres de reproduction}

\section{Prolificité}

La prolificité moyenne a été plus élevée pour la chèvre rousse et pour chaque rang de mise bas (tableau II). Néanmoins, le test de Student n'a pas montré de différence significative entre la chèvre rousse et la chèvre noire pour ce paramètre. Aussi, l'analyse de

\section{Tableau I}

Nombre d'animaux par groupe d'observations et par arrondissement

\begin{tabular}{|c|c|c|c|c|c|c|c|c|c|c|c|c|c|c|c|}
\hline \multirow[t]{2}{*}{$\begin{array}{l}\text { Paramètres } \\
\text { (lieu) }\end{array}$} & \multicolumn{3}{|c|}{ Prolificité } & \multicolumn{3}{|c|}{ Age $1^{\text {ère }}$ mise bas } & \multicolumn{3}{|c|}{$\begin{array}{l}\text { Intervalles } \\
\text { entre mises bas }\end{array}$} & \multicolumn{3}{|c|}{$\begin{array}{l}\text { Mensurations } \\
\text { et poids }\end{array}$} & \multicolumn{3}{|c|}{ Croissance } \\
\hline & $\mathbf{N}$ & $\mathbf{R}$ & Total & $\mathbf{N}$ & $\mathbf{R}$ & Total & $\mathbf{N}$ & $\mathbf{R}$ & Total & $\mathbf{N}$ & $\mathbf{R}$ & Total & $\mathbf{N}$ & $\mathbf{R}$ & Total \\
\hline G.-Roumdji & 13 & 12 & 25 & 12 & 11 & 23 & 12 & 12 & 24 & 13 & 13 & 26 & 8 & 7 & 15 \\
\hline Madarounfa & 9 & 13 & 22 & 7 & 7 & 14 & 8 & 8 & 16 & 9 & 13 & 22 & 12 & 11 & 23 \\
\hline Tessaoua & 17 & 21 & 38 & 12 & 12 & 24 & 17 & 19 & 36 & 17 & 21 & 38 & 0 & 0 & 0 \\
\hline Total & 39 & 46 & 85 & 31 & 30 & 61 & 37 & 39 & 76 & 39 & 47 & 86 & 20 & 18 & 38 \\
\hline
\end{tabular}

$\mathrm{N}$ : caprins noirs ; $\mathrm{R}$ : caprins roux 
variance a montré que ni le lieu ni la robe n'ont été liés à une différence significative de la prolificité. Les deux races ont été prolifiques, même si elles l'ont moins été que les chèvres naines et celles de Massakory (tableau III). Ce paramètre a été influencé par la taille de la portée. Si aucun cas de naissance triple ou quadruple n'a été enregistré, les naissances doubles ont représenté 24,5 et 36,5 p. 100 des observations totales respectivement chez la chèvre noire et chez la chèvre rousse.

Le tableau IV montre une augmentation de la taille de la portée avec le rang de mise bas. Cette tendance a déjà été signalée par Haumesser sur la chèvre rousse de Maradi (11), Dumas et coll. sur la chèvre de Massakory au Tchad (7) et Gerbaldi sur la chèvre bariolée du Niger (9).
Ainsi, quel qu'ait été le village, la prolificité de la chèvre à robe rousse a été légèrement plus élevée que celle de la chèvre à robe noire. Elle a varié de 121 à 140 p. 100 pour les deux robes confondues.

\section{Intervalles entre mises bas}

Concernant les intervalles entre mises bas moyens des deux races (tableau II), l'analyse statistique n'a pas montré de différence significative au seuil de 5 p. 100. Cet intervalle a été long (supérieur à un an) et supérieur à ce qui a été observé par d'autres auteurs $(9,11,13$, 14) sur d'autres races (tableau V). Ceci peut s'expliquer par l'insuffisance des boucs dans les villages et la pratique de mise au piquet des caprins pendant toute la saison des cultures (juin à novembre).

\section{Tableau II}

Paramètres de reproduction comparatifs de la chèvre rousse et de la chèvre noire

\begin{tabular}{lllll} 
Paramètres & \multicolumn{2}{c}{ Chèvre rousse } & \multicolumn{2}{c}{ Chèvre noire } \\
\hline Prolificité moyenne (\%) & $\mathrm{n}=46$ & 136 & $\mathrm{n}=39$ & 124 \\
Intervalle moyen entre mises bas (jours) & $\mathrm{n}=39$ & 386 & $\mathrm{n}=37$ & 363 \\
Age moyen à la 1ère mise bas (mois) & $\mathrm{n}=30$ & $13,57 \pm 2,5$ & $\mathrm{n}=31$ & $13,68 \pm 2,91$
\end{tabular}

Tableau III

Prolificité de différentes races caprines d'Afrique

\begin{tabular}{lcl} 
Race & Prolificité (\%) & Auteur et année \\
\hline Chèvre rousse & 147 & Haumesser, 1975, Revue Elev. Méd. vét. Pays trop. \\
& 125 & Verhults, 1995, Ivtg \\
Caprin du Sahel tchadien & $107-108$ & Dumas, 1977, Gerdat-lemvt \\
Chèvre bariolée du Niger & 126 & Haumesser, 1975, Revue Elev. Méd. vét. Pays trop. \\
Chèvre naine d'Afrique occidentale & 175 & Kabbali et Berger, 1990, Actes Edition \\
Chèvre de Massakory & 143 & lemvt, 1977
\end{tabular}

\section{Tableau IV}

Type de naissance en fonction du rang de mise bas et de la robe

\begin{tabular}{|c|c|c|c|c|c|c|c|c|}
\hline \multirow{2}{*}{$\begin{array}{l}\text { Rang de } \\
\text { mise bas }\end{array}$} & \multirow[t]{2}{*}{ Robe } & \multirow[t]{2}{*}{ Effectif } & \multicolumn{2}{|c|}{ Naissance double } & \multicolumn{2}{|c|}{ Naissance simple } & \multirow{2}{*}{$\begin{array}{l}\text { Total } \\
\text { cabris }\end{array}$} & \multirow{2}{*}{$\begin{array}{c}\text { Taille } \\
\text { portée }\end{array}$} \\
\hline & & & Nb. & $\%$ & Nb. & $\%$ & & \\
\hline \multirow[t]{2}{*}{1 ère } & Noire & 39 & 3 & 7,7 & 36 & 92,3 & 42 & 1,08 \\
\hline & Rousse & 45 & 5 & 11,1 & 40 & 88,9 & 50 & 1,12 \\
\hline \multirow[t]{2}{*}{$2^{e}$} & Noire & 39 & 12 & 30,8 & 27 & 69,2 & 51 & 1,31 \\
\hline & Rousse & 45 & 17 & 37,8 & 28 & 62,2 & 66 & 1,38 \\
\hline \multirow[t]{2}{*}{$3^{e}$} & Noire & 34 & 10 & 29,4 & 24 & 70,6 & 44 & 1,29 \\
\hline & Rousse & 40 & 20 & 50 & 20 & 50 & 60 & 1,5 \\
\hline \multirow[t]{2}{*}{$4^{e}$} & Noire & 19 & 7 & 38,8 & 12 & 63,2 & 23 & 1,37 \\
\hline & Rousse & 18 & 12 & 66,7 & 6 & 33,3 & 30 & 1,67 \\
\hline \multirow[t]{2}{*}{ Total } & Noire & 131 & 32 & 24,4 & 99 & 75,5 & 163 & 1,24 \\
\hline & Rousse & 148 & 54 & 36,5 & 94 & 63,6 & 202 & 1,36 \\
\hline
\end{tabular}


Tableau V

Intervalle entre mises bas chez quelques races caprines

\begin{tabular}{lcl} 
Race & $\begin{array}{c}\text { Intervalle entre mises bas } \\
\text { (jours) }\end{array}$ & Auteur et année \\
\hline Chèvre rousse & 332 & Haumesser, 1975, Revue Elev. Méd. vét. Pays trop. \\
Chèvre bariolée du Niger & 258 & Gerbaldi, 1978, lemvt \\
Chèvre naine de l'Afrique de l'Ouest & 283 & Kabbali et Berger, 1990, Actes Edition \\
Chèvre Massakory du Tchad & 261 & lemvt, 1977
\end{tabular}

\section{Age moyen à la première mise bas}

Les âges moyens à la première mise bas des femelles des deux couleurs (tableau II) ont été semblables à celui obtenu par Haumesser (11) chez la chèvre rousse en élevage. Il n'a pas été observé de lien entre la couleur de la robe ou le milieu sur l'âge à la première mise bas. Aussi, le test de Student n'a pas différencié la moyenne des chèvres noires de celle des chèvres rousses en milieu villageois. Ces chèvres ont été plus précoces que les chèvres rousses de l'étude de Verhults (20 mois) en 1995 (20) et plus tardives que la chèvre Mossi du Burkina qui avait un âge moyen de 11 mois (3).

\section{Poids et mensurations}

Les poids moyens et mensurations ont été relevés dans les différents arrondissements (tableau VI). Ces résultats ont été semblables à ceux précédemment obtenus par d'autres auteurs $(4,19)$. Le test de Student n'a pas relevé de lien entre la couleur de la robe et le poids et les différentes mensurations. Ceci permet de considérer que les deux chèvres ont la même stature physique. Cependant, on note une supériorité sur tous les paramètres des chèvres des deux couleurs de l'arrondissement de Guidan-Roumdji, liée certainement à la conduite de l'élevage. En effet, la période de mesures ayant coïncidé avec la saison des pluies, les animaux de cet arrondissement étaient conduits au pâturage, pendant que ceux des autres arrondissements restaient au piquet au village dans la majorité des cas.

\section{Croissance}

\section{Evolution pondérale de la naissance à 60 jours}

Les données relatives à la croissance des cabris des deux robes de la naissance à 60 jours ont indiqué que le poids moyen à la naissance a été de $1,95 \pm 0,36 \mathrm{~kg}(\mathrm{n}=20)$ pour les chèvres à robe noire et de $1,82 \pm 0,45(\mathrm{n}=18$ ) pour les chèvres rousses (tableau VII). Il n'y a pas eu de lien entre la couleur de la robe et le poids à la naissance. Cependant, les mâles noirs tendaient à être plus lourds que les mâles roux. Ces poids à la naissance ont été semblables à ceux déjà trouvés chez la chèvre rousse de Maradi, en station comme en élevage, par d'autres auteurs $(4,12,17)$. Les mâles sont apparus légèrement plus lourds que les femelles à la naissance, observation faite antérieurement par Haumesser (11) et Oumara (17) chez la chèvre rousse, Alexandre et coll. (1) chez la chèvre créole.

Le poids moyen à 30 jours des cabris roux a été supérieur à celui des cabris noirs, mais cette différence n'a pas été significative. Le poids moyen des cabris de l'échantillon de la présente étude (noirs et roux) a été de 3,68 $\pm 0,88 \mathrm{~kg}$. Il a été légèrement inférieur aux résultats de Nicolas cité par Robinet (19) et d'Oumara (17), tous deux en station. Cette différence pourrait être inhérente à la période de l'étude, ces auteurs ayant mené leurs travaux en saison sèche humide.

A deux mois également, les cabris roux ont pesé plus que les cabris noirs sans que cette différence n'ait été statistiquement significative.

Tableau VI

Poids et mensurations des femelles selon la robe et I'arrondissement enquêté

\begin{tabular}{|c|c|c|c|c|c|c|c|c|}
\hline & Nb. & $\begin{array}{c}\text { Poids } \\
\text { (kg) }\end{array}$ & $\begin{array}{c}\text { Haut. au } \\
\text { garrot }(\mathrm{cm})\end{array}$ & $\begin{array}{l}\text { Long. de } \\
\text { la tête }(\mathrm{cm})\end{array}$ & $\begin{array}{l}\text { Périmètre } \\
\text { thorac. }(\mathrm{cm})\end{array}$ & $\begin{array}{c}\text { Profondeur } \\
\text { poitrine }(\mathbf{c m})\end{array}$ & $\begin{array}{l}\text { Long. scapulo- } \\
\text { ischiale }\end{array}$ & $\begin{array}{l}\text { Long. scapulo- } \\
\text { iliale }\end{array}$ \\
\hline Noire/GR & 13 & 30,0 & 68,0 & 18,1 & 72,2 & 36,7 & 66,2 & 54,3 \\
\hline Noire/Mdfa & 9 & 25,5 & 64,3 & 18,3 & 69,0 & 33,7 & 61,6 & 51,2 \\
\hline Noire/Tessa & 17 & 27,5 & 64,6 & 18,5 & 69,5 & 36,2 & 64,7 & 52,4 \\
\hline Rousse/GR & 13 & 30,0 & 64,2 & 18,4 & 72,7 & 37,1 & 66,0 & 54,2 \\
\hline Rousse/Mdfa & 13 & 25,7 & 64,8 & 17,8 & 68,9 & 33,2 & 62,6 & 51,4 \\
\hline Rousse/Tessa & 21 & 28,6 & 65,5 & 18,5 & 70,8 & 36,7 & 67,3 & 53,4 \\
\hline Noire & 39 & 27,9 & 65,7 & 18,3 & 70,3 & 35,8 & 64,5 & 52,7 \\
\hline Rousse & 47 & 28,2 & 64,9 & 18,3 & 70,8 & 35,9 & 65,6 & 53,0 \\
\hline Noire et rousse & 86 & 28,1 & 65,3 & 18,3 & 70,6 & 35,9 & 65,1 & 52,9 \\
\hline
\end{tabular}

GR : Guidan-Roumdji ; Mdfa : Madarounfa ; Tessa : Tessaoua 


\section{Tableau VII}

Performances de croissance de cabris selon la robe et le sexe

\begin{tabular}{|c|c|c|c|c|c|c|}
\hline \multirow[t]{2}{*}{ Paramètres } & \multicolumn{2}{|c|}{ Robe } & \multicolumn{2}{|c|}{ Mâle } & \multicolumn{2}{|c|}{ Femelle } \\
\hline & Noire & Rousse & Noire & Rousse & Noire & Rousse \\
\hline Effectif & $\mathrm{n}=21$ & $\mathrm{n}=21$ & $\mathrm{n}=7$ & $\mathrm{n}=8$ & $\mathrm{n}=14$ & $n=13$ \\
\hline \multicolumn{7}{|l|}{ Poids vif (kg) } \\
\hline Naissance & $1,95 \pm 0,36$ & $1,82 \pm 0,45$ & $2,15 \pm 0,33$ & $1,84 \pm 0,54$ & $1,82 \pm 0,34$ & $1,80 \pm 0,28$ \\
\hline A 30 jours & $3,42 \pm 0,88$ & $3,94 \pm 0,81$ & $3,28 \pm 0,84$ & $4,13 \pm 0,68$ & $3,49 \pm 0,88$ & $3,62 \pm 0,95$ \\
\hline A 60 jours & $5,86 \pm 0,59$ & $6,07 \pm 0,56$ & $6,08 \pm 0,40$ & $6,05 \pm 0,46$ & $5,75 \pm 0,66$ & $6,09 \pm 0,68$ \\
\hline \multicolumn{7}{|l|}{$\mathrm{Gmq}^{1}$ (g/jour) } \\
\hline $0-30 \mathrm{j}$ & 49 & 70,7 & 44,7 & 77,7 & 48,7 & 59,3 \\
\hline $30-60$ j & 81,3 & 71 & 86,3 & 65,3 & 82,3 & 81 \\
\hline
\end{tabular}

${ }^{1}$ Gain moyen quotidien

\section{Gains moyens quotidiens}

Les gains moyens quotidiens des chevreaux roux entre 0 et 30 jours ont été de 70,7 g par jour contre $49 \mathrm{~g}$ par jour pour les cabris noirs. Ces données ont été différentes de celles enregistrées sur des chevreaux roux par Oumara (61,33 g par jour) (17) au Centre d'élevage caprin au Niger et par Denis (4) à Dara au Sénégal. La tendance semble s'être un peu inversée pour les gains moyens quotidiens entre 30 et 60 jours : $81,3 \mathrm{~g}$ par jour pour les noirs et $71 \mathrm{~g}$ par jour pour les roux. Globalement, il n'y a pas eu de différence significative entre les deux robes en termes de croissance jusqu'à l'âge de deux mois. L'allure des courbes a été similaire à celle observée chez la chèvre créole dans le cas de portée double (1).

\section{- CONCLUSION}

Cette étude n'a pas mis en évidence de différence significative entre les principales caractéristiques de reproduction, les mensurations corporelles et la croissance de 0 à 60 jours de la chèvre rousse en milieu villageois, et celles de la chèvre noire. Ces résultats apportent quelques éléments d'appréciation sur la parenté des deux caprins, même s'ils ne permettent pas d'établir que la chèvre noire est de même type génétique que la chèvre rousse. Cette étude devrait être poursuivie pour prendre en compte des effectifs plus importants et pour ajouter d'autres paramètres comme la fécondité, la qualité de la peau, la production laitière, etc. Aussi, des études sur le calcul d'une distance génétique sur la base d'outils moléculaires plus précis que les seules performances apporteraient des précisions considérables aux connaissances actuelles.

\section{Remerciements}

Les auteurs remercient M. Lippert, assistant technique Belge, et M. Gaya, directeur du Centre caprin de Maradi, pour leur concours, de même que le personnel et les agents vulgarisateurs du projet d'Appui à l'élevage de la chèvre rousse pour leur contribution dans l'exécution des travaux de terrain.

\section{BIBLIOGRAPHIE}

1. ALEXANDRE G., AUMONT G., FLEURY J., MAINAUD J.C., KANDASSAMY T., 1997. Performances zootechniques de la chèvre créole allaitante de Guadeloupe. Bilan de 20 ans dans un élevage expérimental de I'Inra. Prod. Anim., $10:$ 7-20.

2. BEMBELLO A., 1961. La chèvre rousse et son exploitation au Niger. Thèse Doct. vét., Ecole nationale vétérinaire, Toulouse, France, 110 p.

3. CHARRAY J., COULOMB J., HAUMESSER J.B., 1980. Les petits ruminants $d^{\prime}$ Afrique centrale et $d^{\prime}$ Afrique de l'Ouest : synthèse des connaissances actuelles. Maisons-Alfort, France, lemvt, 295 p.

4. DENIS J.P., 1972. Rapport sur les résultats d'introduction de la chèvre rousse de Maradi au Sénégal. Dakar, Sénégal, Lnerv.

5. Direction départementale de l'élevage et des industries animales de Maradi, 1999. Rapport annuel d'activités. Maradi, Niger, Ddeia, 59 p.

6. DUMAS R., 1977. Etude sur l'élevage des petits ruminants du Tchad. Analyse des caractéristiques de la reproduction. Maisons-Alfort, France, lemvt, 32 p. (Fiche de synthèse $n^{\circ} 5$ )
7. DUMAS R., LEFEVRE P., DESLANDES P., 1977 Etude sur l'élevage des petits ruminants au Tchad. Maisons-Alfort, France, Gerdat-lemvt, 354 p.

8. DUMAS R., RAYMOND H., 1974. L'élevage des petits ruminants dans les circonscriptions de Kaya, Ouahigouya et du Sahel. Paris, France, Société pour le développement économique et social.

9. GERBALDI P., 1978. Divers rapports petits ruminants du Niger. Maisons-Alfort, France, lemvt.

10. GUILLERMO L., 1948. Chèvre Angora et Mohair à Madagascar. Thèse Doct. vét., Ecole nationale vétérinaire, Maisons-Alfort, France, 99 p.

11. HAUMESSER J.B., 1975. Quelques aspects de la reproduction chez la chèvre rousse de Maradi. Comparaison avec d'autres races tropicales ou subtropicales. Revue Elev. Méd. vét. Pays trop., 28 : 225-234.

12. HAUMESSER J.B., 1975. Modalités et résultats des enquêtes sur les petits ruminants au Niger. Journées techniques Production animale. Maisons-Alfort, France, lemvt, p. 54-60.

13. IEMVT, 1977. La race caprine du Sahel : résultats d'une année d'observations. Rapport annuel du Lrvz de Farcha. Maisons-Alfort, France, lemvt. 
14. KABBALI A., BERGER Y.M., 1990. L'élevage du mouton dans un pays à climat méditerranéen. Casablanca, Maroc, Institut agronomique et vétérinaire Hassan II, Actes Edition, 235 p.

15. Ministère du Plan, 1997. Schéma directeur de développement régional de Maradi, Niger, vol. 1. Maradi, Niger, ministère du Plan, $150 \mathrm{p}$.

16. MOUDY M.S., 1985. La sélection de la chèvre rousse de Maradi : buts, méthodes, résultats et impacts. Rapport de stage. Niamey, Niger, faculté d'Agronomie, $42 \mathrm{p}$.

17. OUMARA A.D., 1986. Croissance et viabilité de la chèvre rousse de Maradi au Centre d'élevage caprin de Maradi (Niger), Thèse Doct. vét., Eismv, Dakar, Sénégal, 122 p. (N²2)

\section{Summary}

Marichatou H., Mamane L., Banoin M., Baril G. Zootechnical Performances of Goats in Niger: Comparative Study of the Maradi Russet Goat with the Black-Coat Goat in Maradi Area

The main objective of this study was not only to contribute to the knowledge of the Maradi russet goat, but also to compare some of its parameters (reproduction, adult weight, measurements, growth) with those of the black goat. Retrospective oral surveys were carried out on three groups of black, and russet goats, in which 106 goats gave birth 3 or 4 times, 97 of them twice and 87 were primiparous. Seven measurements as well as adult weights were recorded in 125 russet, and black females of 4 years \pm 3 months of age. Weight changes in 38 black, and russet kids were monitored from birth to three months of age in village conditions. Data statistical analysis on both coat-type groups showed, overall, similar results in village conditions. Nevertheless, prolificacy in russet goats was systematically higher than that in black goats regardless of parity.

Key words: Maradi russet goat - Kid - Reproductive performance - Growth - Niger.
18. Programme national de reconstitution du cheptel, 1980. Note sur la diffusion de la chèvre rousse de Maradi. Niamey, Niger, ministère de l'Elevage, $13 \mathrm{p}$.

19. ROBINET A.H., 1967. La chèvre rousse de Maradi. Son exploitation et sa place dans l'économie et l'élevage de la République du Niger. Revue Elev. Méd. vet. Pays trop., 20 : 129-186.

20. VERHULTS A., 1995. Appui à la sélection, à la promotion et à la diffusion de la chèvre rousse de Maradi. Anvers, Belgique, Ivtg, 132 p.

Reçu le 27.05.2002, accepté le 09.01.2003

\section{Resumen}

Marichatou H., Mamane L., Banoin M., Baril G. Rendimientos zootécnicos de los caprinos de Níger: estudio comparativo de la cabra roja de Maradi con su pariente de pelaje negro en la zona de Maradi

El objetivo principal del presente estudio fue no sólo el de contribuir al conocimiento de la cabra roja de Maradi, sino igualmente el de comparar los parámetros de reproducción, el peso del adulto, las medidas y el crecimiento de la cabra negra con los de la roja. Se llevaron a cabo encuestas verbales y retrospectivas para tres lotes de cabras (negras y rojas): 106 con 3 o 4 partos, 97 con dos partos y 87 primíparas. Se efectuaron igualmente ocho medidas, incluyendo el peso adulto, en 125 hembras rojas y negras de 4 años \pm 3 meses. Se siguió la evolución ponderal de 38 cabritos negros y rojos en un medio local desde el nacimiento hasta dos meses. El análisis estadístico de los datos sobre los dos pelajes en un medio de pueblo local mostró resultados globalmente análogos. Sin embargo, la prolificidad fue siempre más elevada en las cabras de pelo rojo que en las de pelo negro, cualquiera que fuese el número de partos.

Palabras clave: Cabra rojiza de Maradi - Cabrito - Reproductividad - Crecimiento - Niger. 